\title{
Prognostic Implication of Adrenocortical Response during the Course of Critical IIIness
}

\author{
Jin Hwa Song', Jung Hee Kim², Sang-Min Lee', Jinwoo Lee' \\ Divisions of ${ }^{1}$ Pulmonary and Critical Care Medicine and ${ }^{2}$ Endocrinology and Metabolism, Department of Internal Medicine, Seoul National University \\ Hospital, Seoul National University College of Medicine, Seoul, Korea
}

Background: Critical illness-related corticosteroid insufficiency (CIRCI) and adrenocorticotropic hormone (ACTH)-cortisol dissociation are hormonal conditions frequently observed in patients in the intensive care unit (ICU). The aim of this study was to evaluate the association between ACTH-cortisol dissociation and clinical outcomes of critically ill patients.

Methods: We retrospectively reviewed the medical records of 94 ICU patients who underwent two rapid cosyntropin tests during hospital admission and compared the clinical aspects of patients with and without ACTH-cortisol dissociation. ACTH-cortisol dissociation was defined as plasma ACTH and serum cortisol concentrations of $<22 \mathrm{pmol} / \mathrm{L}$ and $>600 \mathrm{nmol} / \mathrm{L}$, respectively.

Results: Dissociation was present in 30 of the 94 patients (31.9\%). Patients with ACTH-cortisol dissociation in the initial test had significantly higher hospital mortality rate than those in the control group ( $55 \%$ vs. $25.7 \%, \mathrm{P}=0.013$ ) There was no difference in hospital mortality between patients classified as having $\mathrm{CIRCl}$ and those who were not. In multivariate adjusted Cox regression analysis, the mortality risk was higher in the group with ACTH-cortisol dissociation (hazard ratio, 2.98; 95\% confidence interval, 1.34 to $6.63 ; \mathrm{P}=0.007$ ). Patients with dissociation in two consecutive stimulation tests showed the highest hospital mortality rate among groups classified according to stimulation test results (100\% vs. $31.3 \%)$.

Conclusions: The hospital mortality was higher in ICU patients diagnosed with ACTH-cortisol dissociation. It is clinically feasible to evaluate the presence of ACTH-cortisol dissociation by analyzing rapid ACTH stimulation test results in critically ill patients.

Key Words: adrenocorticotropic hormone; critical illness; mortality; steroids

\section{INTRODUCTION}

In 2017, a multispecialty task force of the Society of Critical Care Medicine (SCCM) and the European Society of Intensive Care Medicine (ESICM) defined the term "critical illness-related corticosteroid insufficiency (CIRCI)." CIRCI refers to the dysregulation of the hypothalamic-pituitary-adrenal axis, altered cortisol metabolism, and peripheral tissue resistance to glucocorticoids. Although the cortisol level is elevated due to decreased cortisol clearance, the target tissue resistance to cortisol is caused by inadequate glucocorticoid receptor alpha activity [1]. The SCCM and ESICM task force in 2017 also proposed that the following conditions may be used as the diagnostic criteria for CIRCI [2]: a delta cortisol (change in baseline

\section{Original Article}

Received: November 8, 2018 Revised: December 29, 2018 Accepted: January 10, 2019

\section{Corresponding author} Jinwoo Lee

Division of Pulmonary and Critical Care Medicine, Department of Internal Medicine, Seoul National University Hospital, Seoul National University College of Medicine, 101 Daehak-ro, Jongno-gu, Seoul 03080, Korea

Tel: +82-2-2072-7593

Fax: +82-2-762-9662

E-mail: realrain7@gmail.com

Copyright @ 2019 The Korean Society of Critical Care Medicine

This is an Open Access article distributed under the terms of Creative Attributions Non-Commercial License (http:// creativecommons.org/li-censes/by-nc/4.0/) which permits unrestricted noncommercial use, distribution, and reproduction in any medium, provided the original work is properly cited. 
cortisol at 60 minutes $)$ of $<9 \mu \mathrm{g} / \mathrm{dl}$ after cosyntropin $(250 \mu \mathrm{g})$ stimulation and a random plasma cortisol of $<10 \mu \mathrm{g} / \mathrm{dl}$.

Previous studies have reported a CIRCI prevalence of $30 \%-$ $70 \%$ in critically ill patients [3-5]. CIRCI in these patients is accompanied by elevated levels of inflammatory markers and coagulopathy and is associated with prolonged intensive care unit (ICU) stay and higher ICU mortality [2]. Although the patients in previous studies were heterogeneous and the CIRCI diagnostic criteria were not unified, the clinical outcomes of critically ill patients with inadequate cortical response were worse than those in patients with an adequate response [3,5-7]. A low random plasma cortisol level could be considered indicative of CIRCI, but the association between cortisol level and clinical outcome was not consistent in previous studies [8-10].

Adrenocorticotropic hormone (ACTH)-cortisol dissociation refers to elevated cortisol levels and low ACTH levels. A decrease in cortisol clearance leads to a decrease in ACTH due to negative inhibitory feedback, which may occur in the chronic phase among critically ill patients. ACTH-cortisol dissociation has been reported in ICU patients [11]; however, to our knowledge, no study has assessed whether it predicts the clinical outcome of critically ill patients as an indicator of abnormal adrenocortical response, such as CIRCI. We attempted to identify changes in the appearance of adrenocortical dissociation and CIRCI in patients who underwent more than one ACTH stimulation test. Therefore, we investigated whether ACTH-cortisol dissociation can predict clinical outcomes in critically ill patients and evaluated the dynamic correlation between two consecutive rapid ACTH stimulation tests.

\section{MATERIALS AND METHODS}

\section{Study Population and Cosyntropin Stimulation Test}

We retrospectively analyzed the medical information of patients admitted the ICUs of a tertiary university hospital between 2006 and 2018. These ICUs included surgical, medical, and emergency ICUs. The study included 104 critically ill patients who underwent two rapid cosyntropin stimulation tests during hospitalization, all of whom underwent the first test during ICU admission. Cosyntropin stimulation test was performed in patients with suspected relative adrenal insufficiency (RAI). Since there are no definite diagnostic criteria for RAI, stimulation test was performed when the clinician suspected the disease. Usually the affected patients had clinical signs such as hyponatremia or refractory shock, fever or general weakness. The test was conducted in the following method. Basal ACTH and cortisol levels were measured by collect-

\section{KEY MESSAGES}

- Adrenocortical dissociation in critically ill patients was related to the worse clinical outcome.

ing the blood sample of the patient before the injection of cosyntropin. The blood used for ACTH measurement was collected in a pre-chilled lithium-heparin tube and carried in an ice box for minimizing degradation of ACTH. The patient was injected intravenously with $250 \mu \mathrm{g}$ of tetracosactrin (Synacthen). After 30 and 60 minutes after the injection of the drug, the patient's blood sample was collected separately and the cortisol level was measured.

\section{Data Collection and Processing}

Demographic data including age, sex, and body mass index were collected. The clinical variables included comorbidities, vital signs at the time of admission to the ICU, and blood test results. The severity of patient illness was assessed by Acute Physiology and Chronic Health Evaluation (APACHE) II score on the first day of ICU admission based on vital signs and laboratory results. The management in the ICU included invasive mechanical ventilation (MV), continuous renal replacement therapy (CRRT), and use of vasoactive agents. We also investigated the presence of septic shock and bacteremia at the time of the cosyntropin stimulation test and whether the patient had acquired a nosocomial infection during their ICU stay. Information was collected to determine if there was a history of steroid use and whether the patient received etomidate before ICU admission. The clinical outcomes of the patients included 30-day ICU mortality, ICU mortality, in-hospital mortality, ICU length of stay (LOS), and hospital LOS. This study was approved by the Institutional Review Board of Seoul National University Hospital (IRB No. 1802-006-918) and patient consent was waived with retrospective design of the study.

Adrenocortical dissociation refers to a condition in which the ACTH concentration is decreased while the cortisol concentration is increased. There remains no consensus regarding the cutoff value to define this condition; therefore, the definition of adrenocortical dissociation used in the present study was an ACTH concentration of $<22 \mathrm{pmol} / \mathrm{L}(100 \mathrm{pg} / \mathrm{ml})$, 25th percentile and cortisol concentration $>600 \mathrm{nmol} / \mathrm{L}(21.75 \mu \mathrm{g} / \mathrm{dl})$, 75th percentile of total population. We compared the clinical outcomes of patients who were evaluated as having adrenocortical dissociation and patients without dissociation in the first cosyntropin stimulation test. 


\section{Statistical Analysis}

Statistical analyses were performed using STATA ver. 13.0 (StataCorp., College Station, TX, USA). Categorical variables were described as number (percent) and continuous variables as mean and standard deviation. For univariate analysis, continuous variables were assessed by Student t-tests, while categorical variables were assessed by Pearson chi-square or Fisher exact tests. Patient survival in the hospital was compared using Kaplan-Meier analysis and log-rank tests. Cox regression analysis was performed for the multivariate analysis of factors affecting hospital mortality. The P-values $<0.05$ were considered statistically significant.

\section{RESULTS}

Among patients admitted to the medical, surgical, emergency, or cardiopulmonary ICUs of a tertiary hospital between 2006 and 2018, a total of 104 patients underwent two cosyntropin stimulation tests during their hospital stays. Among these pa-

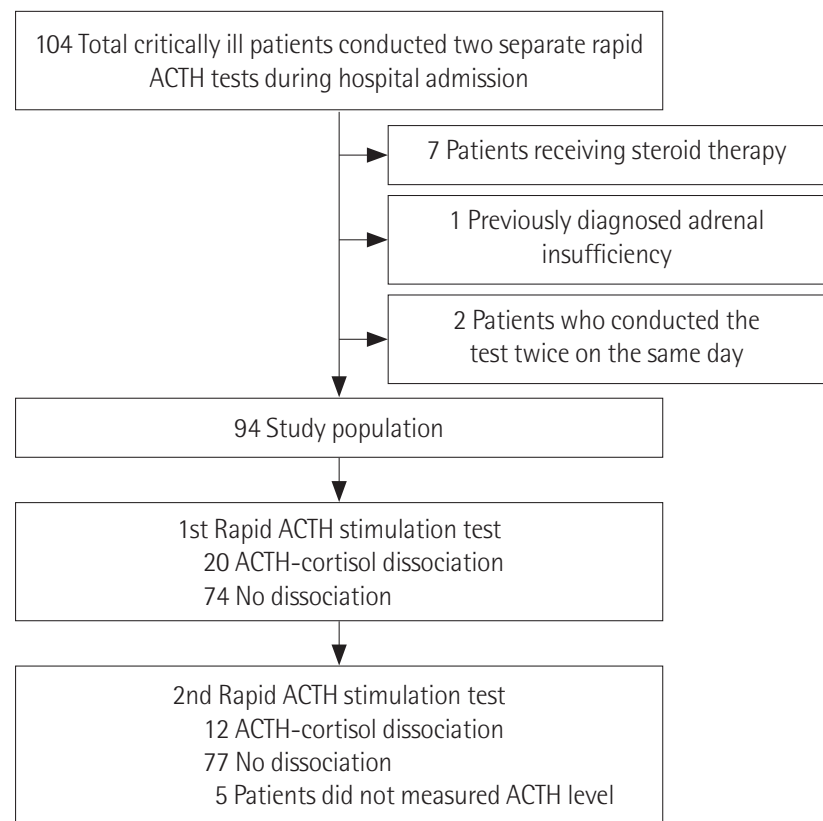

Figure 1. Study population selection flow. ACTH: adrenocorticotropic hormone.

Table 1. Clinical characteristics of the study population

\begin{tabular}{|c|c|c|c|c|}
\hline Variable & Total patients $(n=94)$ & Hospital survivor $(n=64)$ & Hospital non-survivor $(n=30)$ & P-value \\
\hline Female sex & $31(33.0)$ & $24(37.5)$ & $7(23.3)$ & 0.173 \\
\hline Age (yr) & $64.6 \pm 16.1$ & $65.4 \pm 16.4$ & $64.2 \pm 16.0$ & 0.745 \\
\hline BMI $\left(\mathrm{kg} / \mathrm{m}^{2}\right)^{\mathrm{a}}$ & $19.6 \pm 8.0$ & $18.9 \pm 8.1$ & $21.3 \pm 7.7$ & 0.194 \\
\hline $\begin{array}{l}\text { Comorbidity } \\
\text { Malignancy } \\
\text { Chronic liver disease } \\
\text { Diabetes } \\
\text { Hypertension }\end{array}$ & $\begin{array}{c}36(38.3) \\
8(8.5) \\
19(20.2) \\
27(28.7)\end{array}$ & $\begin{array}{c}22 / 64(34.5) \\
4(6.3) \\
12(18.8) \\
11(17.2)\end{array}$ & $\begin{array}{r}14(46.7) \\
4(13.3) \\
7(23.3) \\
16(53.3)\end{array}$ & $\begin{array}{r}0.253 \\
0.251 \\
0.606 \\
<0.001\end{array}$ \\
\hline Serum albumin (g/dl) & $2.6 \pm 0.6$ & $2.6 \pm 0.5$ & $2.6 \pm 0.7$ & 0.931 \\
\hline Serum creatinine (mg/dl) & $1.7 \pm 1.5$ & $1.5 \pm 1.5$ & $2.0 \pm 1.6$ & 0.142 \\
\hline Admission APACHE II score & $24.8 \pm 8.3$ & $24.4 \pm 8.4$ & $25.8 \pm 8.1$ & 0.446 \\
\hline Septic shock & $50(53.2)$ & $32(50.0)$ & $18(60.0)$ & 0.365 \\
\hline Bacteremia & 18 (19.2) & $12(18.8)$ & $6(20.0)$ & 0.886 \\
\hline ICU-acquired infection $^{b}$ & $19(20.2)$ & $4 / 28(14.3)$ & $15 / 66(22.7)$ & 0.351 \\
\hline Previous steroid use & 30 (31.9) & $20(31.3)$ & $10(33.3)$ & 0.840 \\
\hline Previous etomidate use & $24(25.5)$ & $16(25.0)$ & $8(26.7)$ & 0.863 \\
\hline MV & 87 (92.6) & $58(90.6)$ & 29 (96.7) & 0.298 \\
\hline CRRT & $29(30.9)$ & $15(23.4)$ & $14(46.7)$ & 0.023 \\
\hline $\begin{array}{l}\text { Type of ICU } \\
\text { MICU } \\
\text { SICU } \\
\text { CPICU } \\
\text { EICU }\end{array}$ & $\begin{array}{c}41(43.6) \\
11(11.7) \\
1(1.1) \\
41(43.6)\end{array}$ & $\begin{array}{c}28(68.3) \\
8(72.7) \\
0 \\
28(68.3)\end{array}$ & $\begin{array}{c}13(31.7) \\
3(27.2) \\
1(100) \\
13(31.7)\end{array}$ & 0.741 \\
\hline
\end{tabular}

Values are presented as number (\%) or mean \pm standard deviation.

BMI: body mass index; APACHE: Acute Physiology and Chronic Health Evaluation; ICU: intensive care unit; MV: mechanical ventilation; CRRT: continuous renal replacement therapy; MICU: medical ICU; SICU: surgical ICU; CPICU: cardiopulmonary ICU; EICU: emergency ICU.

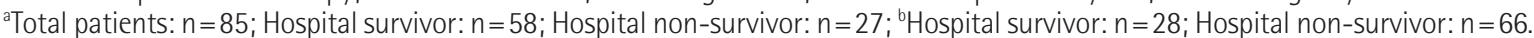


tients, seven with current steroid replacement therapy, one with a previous diagnosis of adrenal insufficiency, and two patients who underwent the test on the same day were excluded. Finally, 94 critically-ill patients were included in the analysis (Figure 1).

\section{Patient Baseline and Clinical Characteristics}

Table 1 shows the baseline and clinical characteristics of these 94 patients. Their mean age was $64.6 \pm 16.1$ years and $33 \%$ were female. The mean APACHE II score was $25.0 \pm 8.3$ and
$53.2 \%$ of the patients had septic shock. Among 87 total patients requiring MV, $19.5 \%$ of patients $(n=17)$ were administered etomidate during rapid sequence intubation, and $80.5 \%$ $(\mathrm{n}=70)$ cases used midazolam during the intubation procedure. During ICU admission, $92.6 \%$ of the patients required invasive MV and 30.9\% received CRRT. Patients with hypertension showed higher hospital mortality (17.2\% vs. $53.3 \%$, $\mathrm{P}<0.001$ ) and patients who received CRRT also showed higher mortality (23.4\% vs. $46.7 \%, \mathrm{P}=0.023)$.

Table 2. Adrenocortical responses of the study population in cosyntropin stimulation tests

\begin{tabular}{|c|c|c|c|c|}
\hline Variable & $\begin{array}{l}\text { Total patients } \\
\qquad(n=94)\end{array}$ & $\begin{array}{l}\text { Hospital survivor } \\
\qquad(n=64)\end{array}$ & $\begin{array}{l}\text { Hospital non-survivor } \\
\qquad(\mathrm{n}=30)\end{array}$ & P-value \\
\hline Day from admission to the 1st test & $18.0 \pm 24.9$ & $16.1 \pm 21.8$ & $21.9 \pm 30.6$ & 0.296 \\
\hline ACTH baseline (pmol/L) & $27.2 \pm 94.6$ & $19.5 \pm 44.9$ & $43.4 \pm 154.6$ & 0.256 \\
\hline Cortisol baseline (nmol/dl) & $565.8 \pm 561.2$ & $530.9 \pm 580.0$ & $640.3 \pm 520.3$ & 0.381 \\
\hline Cortisol after 30 minutes (nmol/dl) & $802.6 \pm 617.5$ & $794.5 \pm 649.3$ & $820.0 \pm 553.5$ & 0.853 \\
\hline Cortisol after 60 minutes (nmol/dl) & $841.5 \pm 561.0$ & $822.2 \pm 568.8$ & $882.7 \pm 551.0$ & 0.629 \\
\hline$\Delta \operatorname{Max}($ cortisol) $(\mathrm{nmol} / \mathrm{dl})$ & $309.9 \pm 422.6$ & $336.8 \pm 482.7$ & $252.7 \pm 248.0$ & 0.371 \\
\hline Cortisol baseline/serum albumin (nmol/mg) & $2.2 \pm 2.3$ & $2.1 \pm 2.6$ & $2.6 \pm 2.5$ & 0.269 \\
\hline Cortisol after 30 minutes/serum albumin (nmol/mg) & $3.1 \pm 2.5$ & $3.1 \pm 2.5$ & $3.3 \pm 2.6$ & 0.619 \\
\hline Cortisol after 60 minutes/serum albumin (nmol/mg) & $3.3 \pm 2.4$ & $3.2 \pm 2.2$ & $3.6 \pm 2.6$ & 0.438 \\
\hline$\Delta \operatorname{Max}($ cortisol)/serum albumin (nmol/mg) & $1.2 \pm 1.6$ & $1.3 \pm 1.8$ & $1.0 \pm 1.1$ & 0.414 \\
\hline Day from admission to the 2 nd test & $49.3 \pm 62.7$ & $47.3 \pm 58.1$ & $53.7 \pm 72.6$ & 0.645 \\
\hline ACTH baseline (pmol/L) ${ }^{\mathrm{a}}$ & $11.3 \pm 12.2$ & $12.1 \pm 12.5$ & $44.8 \pm 53.0$ & 0.419 \\
\hline Cortisol baseline (nmol/dl) & $399.6 \pm 205.1$ & $344.6 \pm 184.1$ & $517.0 \pm 200.9$ & $<0.001$ \\
\hline Cortisol after 30 minutes (nmol/dl) & $603.5 \pm 245.5$ & $567.6 \pm 231.6$ & $680.0 \pm 260.4$ & 0.038 \\
\hline Cortisol after 60 minutes (nmol/dl) & $694.4 \pm 421.7$ & $679.3 \pm 474.9$ & $726.5 \pm 280.8$ & 0.616 \\
\hline$\Delta \operatorname{Max}($ cortisol) $(\mathrm{nmol} / \mathrm{dl})$ & $300.6 \pm 417.6$ & $338.2 \pm 474.0$ & $220.3 \pm 247.7$ & 0.204 \\
\hline Cortisol baseline/serum albumin (nmol/mg) & $1.5 \pm 0.9$ & $1.2 \pm 0.7$ & $2.0 \pm 1.1$ & $<0.001$ \\
\hline Cortisol after 30 minutes/serum albumin (nmol/mg) & $2.2 \pm 1.0$ & $2.0 \pm 0.9$ & $2.6 \pm 1.3$ & 0.013 \\
\hline Cortisol after 60 minutes/serum albumin (nmol/mg) & $2.6 \pm 2.1$ & $2.5 \pm 2.3$ & $2.8 \pm 1.4$ & 0.527 \\
\hline$\Delta \operatorname{Max}($ cortisol)/serum albumin (nmol/mg) & $1.1 \pm 2.0$ & $1.3 \pm 2.3$ & $0.8 \pm 1.0$ & 0.279 \\
\hline
\end{tabular}

Values are presented as mean \pm standard deviation.

ACTH: adrenocorticotropic hormone.

Total patients: $n=89$; Hospital survivor: $n=59$.

Table 3. Clinical outcomes in groups with ACTH-cortisol dissociation in the first cosyntropin stimulation test

\begin{tabular}{lcccc}
\hline Variable & $\begin{array}{c}\text { Total patients } \\
(\mathrm{n}=94)\end{array}$ & $\begin{array}{c}\text { ACTH-cortisol dissociation } \\
(\mathrm{n}=20)\end{array}$ & $\begin{array}{c}\text { No dissociation } \\
(\mathrm{n}=74)\end{array}$ & P-value \\
\hline Hospital mortality & $30 / 94(31.9)$ & $11 / 20(55.0)$ & $19 / 74(25.7)$ & 0.013 \\
28-Day mortality & $6 / 58(10.3)$ & $3 / 10(30.0)$ & $3 / 48(8.3)$ & 0.025 \\
90-Day mortality & $14 / 90(15.6)$ & $7 / 19(36.8)$ & $9 / 71(12.7)$ & 0.014 \\
ICU mortality & $13 / 37(35.1)$ & $7 / 10(70.0)$ & $6 / 27(22.2)$ & 0.017 \\
Hospital LOS & $113.9 \pm 138.6$ & $90.9 \pm 106.4$ & $120.2 \pm 146.1$ & 0.406 \\
ICU LOS & $31.0 \pm 55.3$ & $34.1 \pm 47.8$ & $30.2 \pm 57.4$ & 0.780 \\
\hline
\end{tabular}

Values are presented as number/total number (\%) or mean \pm standard deviation.

ACTH: adrenocorticotropic hormone; ICU: intensive care unit; LOS: length of stay. 


\section{Adrenocortical Responses of Study Population in Cosyntropin Stimulation Tests}

We also compared the rapid ACTH stimulation test results of survivors and non-survivors. There was no significant difference in ACTH and cortisol basal levels between the two groups when comparing the first test. However, in the second test, the baseline cortisol level and the cortisol level after 30 minutes for the non-survivor group were statistically significantly higher. Similar results were obtained when comparing cortisol levels after correcting for serum albumin levels (Table 2).

\section{Effect of ACTH-Cortisol Dissociation on Clinical Outcomes} We compared the clinical outcome between patients with and without ACTH cortisol dissociation in first stimulation test, as shown in Table 3. The dissociation and no-dissociation groups had similar demographic and clinical characteristics (Supplementary Table 1), the overall hospital mortality was $31.9 \%$ and

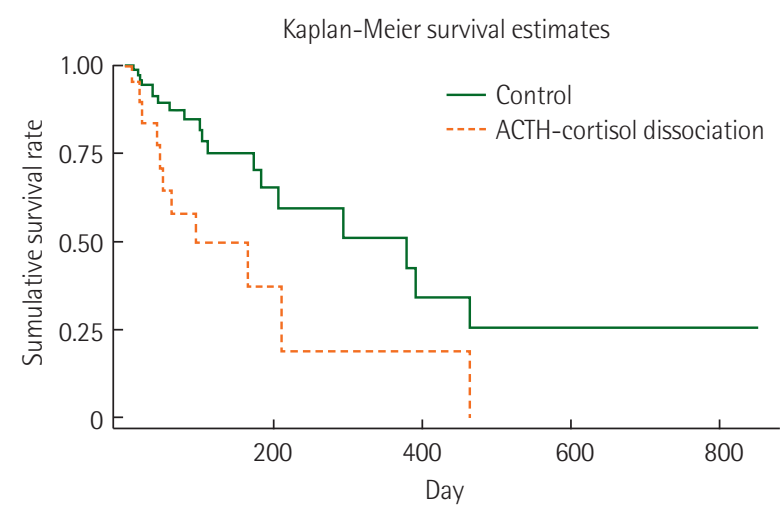

Figure 2. Kaplan-Meier survival analysis of patients. Adrenocorticotropic hormone (ACTH)-cortisol dissociation group refers to critically-ill patients with ACTH less than $22 \mathrm{pmol} / \mathrm{L}$ and cortisol greater than $600 \mathrm{nmol} / \mathrm{L}$ in the first rapid Cosyntropin stimulation test and control denotes that groups of critically ill patients without ACTH-cortisol dissociation in the first test. the ICU mortality rate was $14.9 \%$ (14/94, data not shown). The hospital mortality rates were $55 \%$ and $25.7 \%$ in the dissociation and control groups, respectively. The mortality rate was higher in the dissociation group compared to the 28-day mortality rate only in patients who underwent two tests within 28 days. There was no difference in hospital LOS between the two groups although the mean value of the dissociation group was lower. The 90-day mortality rate in patients who underwent two cosyntropin tests within 90 days was also higher in the dissociation group and consistent with the analysis of patients who underwent two cosyntropin tests during the ICU period (Table 3). These results were consistent in patients without etomidate (Supplementary Table 2).

We also compared the clinical outcomes of the CIRCI group defined by the 2017 SCCM/ESICM criteria but found no statistically significant difference. The outcomes were similar between patients with decreased and increased cortical response in the second test (Supplementary Table 3). KaplanMeier time series analysis showed a higher mortality rate in the dissociation group than that in the control group ( $P=0.009$, log-rank test) (Figure 2). A Cox regression univariate analysis of factors determining hospital mortality revealed that ACTH-cortisol dissociation was associated with higher hospital mortality. Furthermore, ACTH-cortisol dissociation was significantly associated with higher hospital mortality in multivariate analysis adjusted for age, sex, presence of cancer, APACHE II score, hypertension and medical history of malignancy (Table 4).

\section{Differences in Clinical Outcomes According to Changes in ACTH-Cortisol Dissociation in Cosyntropin Stimulation Tests}

To analyze the effect of ACTH-cortisol dissociation on clinical prognosis, we divided the patients into four groups. The first

Table 4. Cox regression analysis of hospital mortality

\begin{tabular}{|c|c|c|c|c|}
\hline \multirow{2}{*}{ Variable } & \multicolumn{2}{|c|}{ Univariate analysis } & \multicolumn{2}{|c|}{ Multivariate analysis } \\
\hline & $\mathrm{HR}(95 \% \mathrm{Cl})$ & P-value & $\mathrm{HR}(95 \% \mathrm{Cl})$ & P-value \\
\hline Female sex & $0.53(0.23-1.24)$ & 0.144 & $1.05(0.38-2.89)$ & 0.930 \\
\hline Age & $1.00(0.99-1.03)$ & 0.566 & $0.98(0.96-1.01)$ & 0.202 \\
\hline ACTH-cortisol dissociation & $2.55(1.21-5.38)$ & 0.014 & $3.18(1.54-6.96)$ & 0.004 \\
\hline Septic shock & $1.66(0.80-3.47)$ & 0.174 & $1.99(0.88-4.54)$ & 0.099 \\
\hline APACHE II score & $1.02(0.98-1.06)$ & 0.374 & $1.04(0.98-1.10)$ & 0.184 \\
\hline Malignancy & $1.63(0.79-3.35)$ & 0.188 & $3.20(1.30-7.82)$ & 0.011 \\
\hline Hypertension & $2.24(1.08-4.66)$ & 0.031 & $3.74(1.43-9.73)$ & 0.007 \\
\hline
\end{tabular}

HR: hazard ratio; Cl: confidence interval; ACTH: adrenocorticotropic hormone; APACHE: Acute Physiology and Chronic Health Evaluation. 
Table 5. Differences in clinical outcomes among groups classified according to changes in ACTH-cortisol dissociation status

\begin{tabular}{|c|c|c|c|c|c|}
\hline \multirow{2}{*}{ Variable } & \multicolumn{5}{|c|}{ Analyzed patients $(n=89)$} \\
\hline & Group $1(n=62)$ & Group $2(n=3)$ & Group $3(n=15)$ & Group $4(n=9)$ & P-value \\
\hline Test interval (day) & $34.5 \pm 59.0$ & $5.3 \pm 3.2$ & $30.5 \pm 23.6$ & $28.9 \pm 16.1$ & 0.120 \\
\hline Hospital mortality & $15 / 62(24.2)$ & $3 / 3(100.0)$ & $8 / 15(53.3)$ & $4 / 9(44.4)$ & 0.009 \\
\hline 28-Day mortality $(n=54)$ & $3 / 42(7.2)$ & 1/3 (33.3) & 2/6 (33.3) & $0 / 3$ & 0.136 \\
\hline 90-Day mortality $(n=88)$ & $6 / 61(10.3)$ & $3 / 3(100.0)$ & $4 / 15(26.7)$ & $1 / 9(11.1)$ & $<0.001$ \\
\hline ICU mortality $(n=34)$ & $4 / 20(20.0)$ & $3 / 3(100.0)$ & $4 / 7(57.1)$ & $2 / 4(50.0)$ & 0.030 \\
\hline Hospital LOS & $114.8 \pm 142.4$ & $37.3 \pm 14.6$ & $106.9 \pm 118.2$ & $171.0 \pm 184.0$ & 0.579 \\
\hline ICU LOS & $21.8 \pm 22.6$ & $26.3 \pm 8.1$ & $38.5 \pm 54.6$ & $83.7 \pm 147.7$ & 0.607 \\
\hline
\end{tabular}

Values are presented as mean \pm standard deviation or number/total number (\%).

ACTH: adrenocorticotropic hormone; Group 1: no dissociations in both tests; Group 2: ACTH-cortisol dissociation in both tests; Group 3: ACTH-cortisol dissociation in the first test; Group 4: ACTH-cortisol dissociation in the second test; ICU: intensive care unit; LOS: length of stay.

group consisted of patients without ACTH-cortisol dissociation in both tests, the second group showed dissociations in both tests, the third group showed dissociation in the first test only, and the fourth group showed dissociation in only the second test. Three patients had ACTH-cortisol dissociation in both cosyntropin stimulation tests (group 2); they comprised $3.2 \%$ of the total patient population and had $100 \%$ hospital mortality. Fifteen patients showed dissociation in the only first test (group 3) and had a hospital mortality rate of 53.3\%. The patients with dissociation in the second test (group 4), had a $44.4 \%$ in-hospital mortality. Groups 1, 3, and 4 had an overall hospital mortality rate of $31.3 \%$. The hospital mortality rate of patients without dissociation in two tests was the lowest, at $24.2 \%$. These differences between groups were similar for 90-day and ICU mortality (Table 5).

\section{DISCUSSION}

The present study investigated the relationship between adrenocortical dissociation and hospital mortality in critically ill patients. Adrenocortical dissociation, defined as the combined condition of increased cortisol and suppressed ACTH levels, was associated with increased in-hospital mortality. Every participant in this study underwent two separate cosyntropin stimulation tests during hospitalization and patients with adrenocortical dissociation at least one of the two tests showed worse clinical outcome than those in patients without dissociation in both tests. The association between dissociation and hospital mortality was consistent across time-series and multivariate analyses. In addition, the non-survivor group had an increased basal cortisol level in the second stimulation test. This dissociation occurred during critical illness and was sustained for more than 1 week [12]. We demonstrated that dissociation, reflecting a prolonged stress response, associated with the prognosis of critically ill patients.

Previous reports on Korean ICUs have reported a RAI rate of $70.6 \%$ among septic shock patients [9] and $43.9 \%$ among critically ill patients [5]. These earlier studies defined RAI as an increase in serum cortisol level of less than $9 \mu \mathrm{g} / \mathrm{dl}$ from the baseline after the injection of $250 \mu \mathrm{g}$ cosyntropin $[5,9]$. The frequency of adrenocortical dissociation in the present study was $21.3 \%$. In addition, the proportions of patients diagnosed with CIRCI according to the SCCM/ESICM criteria were as follows: cortisol increase less than $9 \mu \mathrm{g} / \mathrm{dl}$ after stimulation was $53.2 \%$ with and $16.0 \%$ with less than $10 \mu \mathrm{g} / \mathrm{dl}$ random serum cortisol level. The frequencies of adrenal dysfunction such as RAI, CIRCI, and dissociation vary according to the diagnostic criteria. These findings suggest the usefulness of the assessment of adrenal dysfunction in critically ill patients.

Some limitations should be considered in interpreting the results of this study. The first is the inherent nature of its retrospective design. Second, there was heterogeneity in the patient population. This study included patients without septic shock. We also analyzed patients who entered the medical, surgical, and emergency ICUs. Third, compared to previous studies on the effect of RAI or CIRCI on the prognosis of critically ill patients $[5,9,13,14]$, the patients included in this study stayed in the ICU for an average of 30 days or longer. Forth, because of the nature of the retrospective study, the detailed use of various medications including steroids were not assessed. Finally, we defined adrenocortical dissociation as the 25th percentile of ACTH and 75th percentile of cortisol in this study population, but these cutoff values require validation in a prospective large-scale study.

The strength of this study was its analysis of the dynamic correlations in patients who underwent two stimulation tests. 
We assessed changes in two tests and, unlike previous studies, did not find any difference in ICU survival or LOS [13]. To our knowledge, this is the first study to compare the clinical outcome of patients diagnosed with CIRCI using the suggested cutoff value of SCCM/ESICM 2017 guideline. There was no significant difference in the clinical prognosis of patients with CIRCI. Adrenocortical dissociation showed a worse outcome; therefore, the ACTH level should also be considered in the clinical interpretation of cosyntropin stimulation test results. In addition, these results can be universally applied to critically ill patients and compared to previous studies in homogeneous patient groups such as those with septic shock, cardiogenic shock, and trauma.

It is unclear whether steroid replacement is effective in patients with adrenocortical dissociation. Steroid resistance in the peripheral tissue may contribute to the increased cortisol level in patients with dissociation, and supplementation of additional systemic steroids in these cases may have consequences. Only $40 \%$ of patients with adrenocortical dissociation underwent steroid replacement and there was no significant difference in in-hospital mortality between dissociation patients who did and did not receive systemic steroids (62.5\% vs. $50.0 \%, \mathrm{P}=0.670$ ). Although there was no significant difference in mortality, it is possible that steroid replacement may have affected the prognosis of these patients.

In conclusion, the results of our retrospective cohort study showed an increased hospital mortality among patients with adreno-cortical dissociation defined as an elevated basal cortisol level and suppressed ACTH level. Although the patients with ACTH-cortisol dissociation in rapid ACTH stimulation tests is associated with poor outcome in our study, validation in a multicenter prospective study is required to confirm these findings of ACTH-cortisol dissociation.

\section{CONFLICT OF INTEREST}

No potential conflict of interest relevant to this article was reported.

\section{ORCID}

Jin Hwa Song https://orcid.org/0000-0002-8161-3970

Jung Hee Kim https://orcid.org/0000-0003-1932-0234

Sang-Min Lee https://orcid.org/0000-0002-1388-9318

Jinwoo Lee https://orcid.org/0000-0003-0958-106X

\section{AUTHOR CONTRIBUTIONS}

Conceptualization: JL, SML. Data curation: JHS. Formal analysis: JHS. Methodology: JL, JHS, JHK, SML. Project administration: JL. Visualization: JHS. Writing - original draft: JHS. Writing - review \& editing: JHS, JL, JHK, SML.

\section{SUPPLEMENTARY MATERIALS}

The online-only supplement data are available with this article online: https://doi.org/10.4266/acc.2018.00339.

\section{REFERENCES}

1. Annane D, Pastores SM, Arlt W, Balk RA, Beishuizen A, Briegel J, et al. Critical illness-related corticosteroid insufficiency (CIRCI): a narrative review from a Multispecialty Task Force of the Society of Critical Care Medicine (SCCM) and the European Society of Intensive Care Medicine (ESICM). Intensive Care Med 2017;43:1781-92.

2. Annane D, Pastores SM, Rochwerg B, Arlt W, Balk RA, Beishuizen A, et al. Guidelines for the diagnosis and management of critical illness-related corticosteroid insufficiency (CIRCI) in critically ill patients (part I): Society of Critical Care Medicine (SCCM) and European Society of Intensive Care Medicine (ESICM) 2017. Crit Care Med 2017;45:2078-88.

3. Rothwell PM, Udwadia ZF, Lawler PG. Cortisol response to corticotropin and survival in septic shock. Lancet 1991;337: 582-3.

4. Freund Y, Jabre P, Mourad J, Lapostolle F, Reuter PG, Woimant $\mathrm{M}$, et al. Relative adrenal insufficiency in critically ill patient after rapid sequence intubation: KETASED ancillary study. J Crit Care 2014;29:386-9.

5. Kwon YS, Kang E, Suh GY, Koh WJ, Chung MP, Kim H, et al. A prospective study on the incidence and predictive factors of relative adrenal insufficiency in Korean critically-ill patients. J Korean Med Sci 2009;24:668-73.

6. de Jong MF, Beishuizen A, Spijkstra JJ, Groeneveld AB. Relative adrenal insufficiency as a predictor of disease severity, mortality, and beneficial effects of corticosteroid treatment in septic shock. Crit Care Med 2007;35:1896-903.

7. Annane D, Bellissant E. Prognostic value of cortisol response in septic shock. JAMA 2000;284:308-9.

8. Pandya U, Polite N, Wood T, Lieber M. Increased total serum random cortisol levels predict mortality in critically ill trauma patients. Am Surg 2014;80:1112-8.

9. Kwon YS, Suh GY, Kang EH, Koh WJ, Chung MP, Kim H, et al. 
Basal serum cortisol levels are not predictive of response to corticotropin but have prognostic significance in patients with septic shock. J Korean Med Sci 2007;22:470-5.

10. Suresh R, Wig N, Panda PK, Jyotsna VP, Chaturvedi PK, Pandey RM. Serum cortisol level in Indian patients with severe sepsis/septic shock. J Emerg Trauma Shock 2017;10:194-8.

11. Raff H, Biru N, Reisinger N, Kramer DJ. Dissociation of ACTH and cortisol in septic and non-septic ICU patients. Endocrine 2017;55:307-10.

12. Boonen E, Van den Berghe G. Cortisol metabolism in critical illness: implications for clinical care. Curr Opin Endocrinol Diabetes Obes 2014;21:185-92.

13. de Jong MF, Beishuizen A, van Schijndel RJ, Girbes AR, Groeneveld AB. Risk factors and outcome of changes in adrenal response to ACTH in the course of critical illness. J Intensive Care Med 2012;27:37-44.

14. Bagate F, Lellouche N, Lim P, Moutereau S, Razazi K, Carteaux $\mathrm{G}$, et al. Prognostic value of relative adrenal insufficiency during cardiogenic shock: a prospective cohort study with longterm follow-up. Shock 2017;47:86-92. 
Supplementary Table 1. Comparison of groups with and without adrenocorticotropic hormone-cortisol dissociation

\begin{tabular}{lcccc}
\hline Variable & Total patients $(n=94)$ & Dissociation $(n=20)$ & No dissociation $(n=74)$ & P-value \\
\hline Sex (female) & $31 / 94(33.0)$ & $6 / 20(30.0)$ & $25 / 74(33.8)$ & 0.749 \\
Age $(\mathrm{yr})$ & $64.6 \pm 16.1$ & $63.4 \pm 19.3$ & $64.9 \pm 15.2$ & 0.712 \\
BMI $\left(\mathrm{kg} / \mathrm{m}^{2}\right)^{\mathrm{a}}$ & $19.6 \pm 8.0$ & $19.1 \pm 9.3$ & $19.8 \pm 7.7$ & 0.744 \\
Comorbidity & & & & $30 / 74(40.5)$ \\
$\quad$ Malignancy & $36 / 94(38.3)$ & $6 / 20(30.0)$ & $7 / 74(9.5)$ & 0.390 \\
Chronic liver disease & $8 / 94(8.5)$ & $1 / 20(5.0)$ & $2.6 \pm 0.5$ & 0.526 \\
Serum albumin (g/dl) & $2.6 \pm 0.6$ & $2.6 \pm 0.8$ & $1.6 \pm 1.5$ & 0.981 \\
Serum creatinine (mg/dl) & $1.7 \pm 1.5$ & $1.8 \pm 1.6$ & $24.0 \pm 8.2$ & 0.603 \\
APACHE II score & $24.8 \pm 8.3$ & $27.7 \pm 8.1$ & $39 / 74(52.7)$ & 0.079 \\
Septic shock & $50 / 94(53.2)$ & $11 / 20(55.0)$ & $13 / 74(17.6)$ & 0.855 \\
Bacteremia & $18 / 94(19.2)$ & $5 / 20(25.0)$ & $16 / 74(21.6)$ & 0.454 \\
ICU-acquired infection & $19 / 94(20.2)$ & $3 / 20(15.0)$ & $22 / 74(29.7)$ & 0.513 \\
Previous steroid use & $30 / 94(31.9)$ & $8 / 20(40.0)$ & $18 / 74(24.3)$ & 0.382 \\
Previous etomidate use & $24 / 94(25.5)$ & $6 / 20(30.0)$ & $69 / 74(93.2)$ & 0.606 \\
MV & $87 / 94(92.6)$ & $18 / 20(90.0)$ & $23 / 74(31.1)$ & 0.624 \\
CRRT & $29 / 94(30.9)$ & $6 / 20(30.0)$ & 0.926 &
\end{tabular}

Values are presented as number/total number (\%) or mean \pm standard deviation.

BMI: body mass index; APACHE: Acute Physiology and Chronic Health Evaluation; ICU: intensive care unit; MV: mechanical ventilation; CRRT: continuous renal replacement therapy.

Total patients: $n=85$; Dissociation: $n=19$; No dissociation: $n=66$.

Supplementary Table 2. Clinical outcomes of ACTH-cortisol dissociation in etomidate-free population

\begin{tabular}{lccc}
\hline Patient not treated with etomidate & ACTH-cortisol dissociation $(n=14)$ & No dissociation $(n=56)$ & P-value \\
\hline Hospital mortality & $8 / 14(57.1)$ & $14 / 56(25.0)$ & 0.020 \\
28-Day mortality $(n=44)$ & $3 / 8(37.5)$ & $2 / 36(5.6)$ & 0.035 \\
90-Day mortality $(n=66)$ & $6 / 14(42.9)$ & $5 / 52(9.6)$ & 0.003 \\
ICU mortality $(n=28)$ & $6 / 8(75.0)$ & $4 / 20(20.0)$ & 0.006 \\
Hospital LOS & $69.1 \pm 65.3$ & $124.1 \pm 153.3$ & 0.196 \\
ICU LOS & $37.5 \pm 56.2$ & $27.9 \pm 46.0$ & 0.506 \\
\hline
\end{tabular}

Values are presented as number/total number (\%) or mean \pm standard deviation.

ACTH: adrenocorticotropic hormone; ICU: intensive care unit; LOS: length of stay. 
Supplementary Table 3. Differences in clinical outcome according to $\mathrm{CIRCl}$ defined by various criteria

\begin{tabular}{|c|c|c|c|}
\hline Variable & $\begin{array}{l}\mathrm{CIRCl} \text { (increase in cortisol level }<248.3 \mathrm{nmol} / \mathrm{L} \\
\text { from baseline) }(n=50)\end{array}$ & $\begin{array}{l}\text { No CIRCI } \\
(n=44)\end{array}$ & P-value \\
\hline Hospital mortality & 16/50 (32.0) & 14/44 (31.8) & 0.985 \\
\hline 28-Day mortality & $6 / 36(16.7)$ & $1 / 22(4.6)$ & 0.235 \\
\hline 90-Day mortality & 8/43 (18.6) & 8/47 (17.0) & 0.844 \\
\hline ICU mortality & $5 / 19(26.4)$ & $8 / 18(44.4)$ & 0.248 \\
\hline Hospital LOS & $116.7 \pm 153.4$ & $110.7 \pm 121.4$ & 0.836 \\
\hline \multirow[t]{2}{*}{ ICU LOS } & $48.1 \pm 82.3$ & $64.4 \pm 77.6$ & 0.538 \\
\hline & $\begin{array}{c}\text { CIRCI (increase in cortisol level }<275.9 \mathrm{nmol} / \mathrm{L} \\
\text { from baseline) }(n=15)\end{array}$ & $\begin{array}{l}\text { No } \mathrm{CIRCl} \\
(n=79)\end{array}$ & \\
\hline Hospital mortality & $2 / 15(13.3)$ & $28 / 79(35.4)$ & 0.132 \\
\hline 28-Day mortality & $0 / 9$ & $7 / 49$ (14.3) & 0.581 \\
\hline 90-Day mortality & $1 / 15(6.7)$ & $15 / 75(20.0)$ & 0.291 \\
\hline ICU mortality & $1 / 5(20)$ & 12/32 (37.5) & 0.638 \\
\hline Hospital LOS & $144.5 \pm 218.4$ & $144.5 \pm 218.4$ & 0.354 \\
\hline \multirow[t]{2}{*}{ ICU LOS } & $47.5 \pm 94.3$ & $27.9 \pm 44.5$ & 0.209 \\
\hline & $\begin{array}{l}\text { Decrease in } \Delta \text { cortisol in the } 2 \text { nd test } \\
\qquad(n=51)\end{array}$ & $\begin{array}{l}\text { Increase in } \Delta \text { cortisol in the } 2 n d \text { test } \\
\qquad(n=43)\end{array}$ & \\
\hline Hospital mortality & $15 / 51(29.4)$ & $15 / 43(34.9)$ & 0.571 \\
\hline 28-Day mortality & $3 / 51(5.9)$ & $4 / 43(9.3)$ & 0.529 \\
\hline 90-Day mortality & 8/51 (15.7) & 8/43 (18.6) & 0.708 \\
\hline ICU mortality $(n=37)$ & $9 / 19(47.3)$ & $4 / 18(22.2)$ & 0.109 \\
\hline Hospital LOS & $108.3 \pm 113.8$ & $120.5 \pm 164.6$ & 0.672 \\
\hline ICU LOS & $32.4 \pm 53.9$ & $29.3 \pm 57.5$ & 0.788 \\
\hline
\end{tabular}

Values are presented as number/total number (\%) or mean \pm standard deviation.

CIRCI: critical illness-related corticosteroid insufficiency; ICU: intensive care unit; LOS: length of stay. 